\title{
FUNDAMENTAL THEOREM OF WIENER CALCULUS
}

\author{
CHULL PARK \\ Dept. of Math. \& Stat. \\ Miami University \\ Oxford, OH 45056

\section{DAVID SKOUG} \\ Dept. of Math. \& Stat. \\ University of Nebraska \\ Lincoln, NE 68588-0323
}

\section{LAWRENCE SMOLOWITZ}

Dept. of Math. \& Comp. Sci.

Hobert \& William Smith College

Geneva, NY 14456

(Received April 12, 1989)

\begin{abstract}
In this paper we define and develop a theory of differentiation in Wiener space $\mathrm{C}[0, \mathrm{~T}]$. We then proceed to establish a fundamental theorem of the integral calculus for $\mathrm{C}[0, \mathrm{~T}]$. First of all, we show that the derivative of the indefinite Wiener integral exists and equals the integrand functional. Secondly, we show that certain functionals defined on $\mathrm{C}[0, \mathrm{~T}]$ are equal to the indefinite integral of their Wiener derivative.
\end{abstract}

KEY WORDS AND PHRASES. Wiener (measure, integral, derivative, absolute continuity), Lebesgue absolute continuity, fundamental theorem.

1980 MATHEMATICS SUBJECT CLASSIFICATION. 28C20, 46 G05.

\section{INTRODUCTION.}

Consider the Wiener measure space $\left(\mathrm{C}[0, \mathrm{~T}], \mathscr{F}^{*}, \mathrm{~m}_{\mathrm{w}}\right)$ where $\mathrm{C}[0, \mathrm{~T}]$ is the space of all continuous functions $\mathrm{x}$ on $[0, \mathrm{~T}]$ vanishing at the origin. For each partition $\tau=\tau_{\mathrm{n}}=\left\{\mathrm{t}_{1}, \cdots, \mathrm{t}_{\mathrm{n}}\right\}$ of $[0, \mathrm{~T}]$ with $0=\mathrm{t}_{0}<\mathrm{t}_{1}<\cdots<\mathrm{t}_{\mathrm{n}}=\mathrm{T}$, let $\mathrm{X}_{\tau}: \mathrm{C}[0, \mathrm{~T}] \rightarrow \mathbb{R}^{\mathrm{n}}$ be

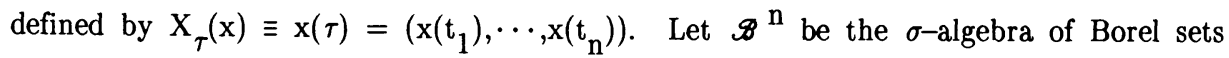
in $\mathbb{R}^{n}$. Then a set of the type 


$$
\mathrm{I}=\left\{\mathrm{x} \in \mathrm{C}[0, \mathrm{~T}]: \mathrm{X}_{\tau}(\mathrm{x}) \in \mathrm{B}\right\} \equiv \mathrm{X}_{\tau}^{-1}(\mathrm{~B}), \mathrm{B} \in \mathscr{B}^{\mathrm{n}}
$$

is called a Wiener interval (or a Borel cylinder). It is well known that

$$
\mathrm{m}_{\mathrm{w}}(\mathrm{I})=\int_{\mathrm{B}} \mathrm{K}(\tau, \vec{\eta}) \mathrm{d} \vec{\eta}
$$

where

$$
\mathrm{K}(\tau, \vec{\eta})=\left\{\prod_{\mathrm{j}=1}^{\mathrm{n}} 2 \pi\left(\mathrm{t}_{\mathrm{j}}-\mathrm{t}_{\mathrm{j}-1}\right)\right\}^{-1 / 2} \exp \left\{-\frac{1}{2} \sum_{\mathrm{j}=1}^{\mathrm{n}} \frac{\left(\eta_{\mathrm{j}}-\eta_{\mathrm{j}-1}\right)^{2}}{\mathrm{t}_{\mathrm{j}}-\mathrm{t}_{\mathrm{j}-1}}\right\}
$$

with $\vec{\eta}=\left(\eta_{1}, \cdots, \eta_{\mathrm{n}}\right)$, and $\eta_{0}=0$. The measure $\mathrm{m}_{\mathrm{w}}$ is a probability measure defined on the algebra $\mathscr{F}$ of all Wiener intervals and $\mathrm{m}_{\mathrm{w}}$ is extended to the Caratheodory extension $\mathscr{I}^{*}$ of $\mathscr{I}$. Let $\mathscr{F}_{\tau}$ be the $\sigma$-algebra generated by the set $\left\{\mathrm{X}_{\tau}^{-1}(\mathrm{~B}): \mathrm{B} \in \mathscr{S}^{\mathrm{n}}\right\}$ with $\tau$ fixed. Then, by the definition of conditional expectation, see Doob [1], Tucker [2] and Yeh [3], for each Wiener integrable function $\mathrm{F}(\mathrm{x})$,

$$
\begin{aligned}
\mu_{\tau}(\mathrm{B}) & \equiv \int_{\mathrm{X}_{\tau}^{-1}(\mathrm{~B})} \mathrm{F}(\mathrm{x}) \mathrm{m}_{\mathrm{w}}(\mathrm{dx})=\int_{\mathrm{X}_{\tau}^{-1}(\mathrm{~B})} \mathrm{E}\left(\mathrm{F} \mid \mathscr{F}_{\tau}\right) \mathrm{m}_{\mathrm{w}}(\mathrm{dx}) \\
& =\int_{\mathrm{B}} \mathrm{E}\left(\mathrm{F}(\mathrm{x}) \mid \mathrm{X}_{\tau}(\mathrm{x})=\vec{\eta}\right) \mathrm{P}_{\mathrm{X}_{\tau}}(\mathrm{d} \vec{\eta}), \mathrm{B} \in \mathscr{B}^{\mathrm{n}},
\end{aligned}
$$

where $\mathrm{P}_{\mathrm{X}_{\tau}}(\mathrm{B})=\mathrm{m}_{\mathrm{w}}\left(\mathrm{X}_{\tau}^{-1}(\mathrm{~B})\right)$, and $\mathrm{E}\left(\mathrm{F}(\mathrm{x}) \mid \mathrm{X}_{\tau}(\mathrm{x})=\vec{\eta}\right)$ is a Lebesgue measurable function of $\vec{\eta}$ which is unique up to null sets in $\mathbb{R}^{\mathrm{n}}$. Also, using (1.1) and (1.3) and choosing $\mathrm{F}(\mathrm{x}) \equiv 1$, we see that

$$
\mathrm{P}_{\mathrm{X}}(\mathrm{d} \vec{\eta})=\mathrm{K}(\tau, \vec{\eta}) \mathrm{d} \vec{\eta}
$$

or

$$
\frac{\mathrm{dP}}{\mathrm{d} \vec{\eta}}=\mathrm{K}(\tau, \vec{\eta}), \vec{\eta} \in \mathbb{R}^{\mathrm{n}}
$$

Next, for each $\mathrm{F} \in \mathrm{L}_{1}\left(\mathrm{C}[0, \mathrm{~T}], \mathrm{m}_{\mathrm{w}}\right)$ and each partition $\tau$ of $\mathrm{C}[0, \mathrm{~T}]$, let

$$
\mathrm{F}_{\tau}=\mathrm{E}\left(\mathrm{F} \mid \mathscr{S}_{\tau}\right)
$$

and

$$
\tilde{\mathrm{F}}(\vec{\eta})=\mathrm{E}\left(\mathrm{F}(\mathrm{x}) \mid \mathrm{X}_{\tau}(\mathrm{x})=\vec{\eta}\right) \equiv \mathrm{E}\left(\mathrm{F} \mid \mathrm{X}_{\tau}\right)(\vec{\eta})
$$

Then, $\left\{\mathrm{F}_{\tau}\right\}$ is a martingale, and by the martingale convergence theorem,

$$
\lim _{\|\tau\| \rightarrow 0} F_{\tau}(x)=F(x)
$$

for almost all $x \in C[0, T]$. Furthermore,

$$
\mathrm{F}(\mathrm{x})=\lim _{\|\tau\| \rightarrow 0} \mathrm{E}\left(\mathrm{F}(\mathrm{y}) \mid \mathrm{X}_{\tau}(\mathrm{y})=\mathrm{x}(\tau)\right)=\lim _{\|\tau\| \rightarrow 0} \tilde{\mathrm{F}}(\mathrm{x}(\tau))
$$

for almost all $\mathrm{x} \in \mathrm{C}[0, \mathrm{~T}]$.

For a given partition $\tau=\tau_{\mathrm{n}}$ of $[0, \mathrm{~T}]$ and $\mathrm{x} \in \mathrm{C}[0, \mathrm{~T}]$, define the polygonal function $[\mathrm{x}] \equiv[\mathrm{x}(\tau)]$ on $[0, \mathrm{~T}]$ by 


$$
[\mathrm{x}](\mathrm{t})=\mathrm{x}\left(\mathrm{t}_{\mathrm{j}-1}\right)+\frac{\mathrm{t}-\mathrm{t}_{\mathrm{j}-1}}{\mathrm{t}_{\mathrm{j}} \mathrm{t}_{\mathrm{j}-1}}\left(\mathrm{x}\left(\mathrm{t}_{\mathrm{j}}\right)-\mathrm{x}\left(\mathrm{t}_{\mathrm{j}-1}\right)\right), \mathrm{t}_{\mathrm{j}-1} \leq \mathrm{t} \leq \mathrm{t}_{\mathrm{j}}, \mathrm{j}=1, \cdots, \mathrm{n} .
$$

Similarly, for each $\vec{\eta}=\left(\eta_{1}, \cdots, \eta_{\mathrm{n}}\right) \in \mathbb{R}^{\mathrm{n}}$, define the polygonal function $[\vec{\eta}]$ of $\vec{\eta}$ on $[0, T]$ by

$$
[\vec{\eta}](\mathrm{t})=\eta_{\mathrm{j}-1}+\frac{\mathrm{t}^{\mathrm{t}} \mathrm{j}_{\mathrm{j}}}{\mathrm{t}_{\mathrm{j}} \mathrm{t}_{\mathrm{j}-1}}\left(\eta_{\mathrm{j}}-\eta_{\mathrm{j}-1}\right), \mathrm{t}_{\mathrm{j}-1} \leq \mathrm{t} \leq \mathrm{t}_{\mathrm{j}}, \mathrm{j}=1, \cdots, \mathrm{n} \text { with } \eta_{0}=0 .
$$

Then both functions $[\mathrm{x}]$ and $[\vec{\eta}]$ are continuous on $[0, \mathrm{~T}]$, their graphs are line segments on each subinterval $\left[\mathrm{t}_{\mathrm{j}-1}, \mathrm{t}_{\mathrm{j}}\right]$, and $[\mathrm{x}]\left(\mathrm{t}_{\mathrm{j}}\right)=\mathrm{x}\left(\mathrm{t}_{\mathrm{j}}\right)$ and $[\vec{\eta}]\left(\mathrm{t}_{\mathrm{j}}\right)=\eta_{\mathrm{j}}$ at each $\mathrm{t}_{\mathrm{j}} \in \tau$.

For $\mathrm{x}, \mathrm{y} \in \mathrm{C}[0, \mathrm{~T}]$, we use the convention:

$$
\mathrm{x} \leq \mathrm{y} \text { if and only if } \mathrm{x}(\mathrm{t}) \leq \mathrm{y}(\mathrm{t}) \text { for every } \mathrm{t} \in[0, \mathrm{~T}]
$$

and

$$
\mathrm{x}<\mathrm{y} \text { if and only if } \mathrm{x}(\mathrm{t})<\mathrm{y}(\mathrm{t}) \text { for every } \mathrm{t} \in(0, \mathrm{~T}] \text {. }
$$

The main purpose of this paper is to define and develop a theory of differentiation in Wiener space $C[0, T]$, and then to establish a fundamental theorem of the integral calculus on $\mathrm{C}[0, T]$; namely, that the Wiener derivative of the indefinite integral $\int_{y \leq x} F(y) m_{w}(d y)$ is $\mathrm{F}(\mathrm{x})$, and that a Wiener absolutely continuous function can be expressed as the indefinite integral of its Wiener derivative. This study was initiated by Smolowitz [4]. In this paper we incorporate some recent results of Park and Skoug [5] to improve and substantially simplify the concepts and results of Smolowitz [4].

\section{THE WIENER DERIVATIVE.}

Our first objective is to define the Wiener derivative $\mathscr{D}_{\mathrm{X}}(\cdot)$ so that

$$
\mathscr{D}_{\mathrm{x}} \int_{\mathrm{y} \leq \mathrm{x}} \mathrm{F}(\mathrm{y}) \mathrm{m}_{\mathrm{w}}(\mathrm{dy})=\mathrm{F}(\mathrm{x})
$$

for $F \in \mathrm{L}_{1}\left(\mathrm{C}[0, \mathrm{~T}], \mathrm{m}_{\mathrm{W}}\right)$. We start by quoting the following theorem from Park and Skoug [5] which plays an important role in this paper.

THEOREM A. Let $\mathrm{F} \in \mathrm{L}_{1}\left(\mathrm{C}[0, \mathrm{~T}], \mathrm{m}_{\mathrm{w}}\right)$. Then for any Borel set $\mathrm{B} \in \mathscr{B}^{\mathrm{n}}$,

where

$$
\mu_{\tau}(\mathrm{B}) \equiv \int_{\mathrm{X}_{\tau}^{-1}(\mathrm{~B})} \mathrm{F}(\mathrm{x}) \mathrm{m}_{\mathrm{w}}(\mathrm{dx})=\int_{\mathrm{B}} \mathrm{E}_{\mathrm{x}}[\mathrm{F}(\mathrm{x})-[\mathrm{x}]+[\vec{\eta}]] \mathrm{P}_{\mathrm{X}_{\tau}}(\mathrm{d} \vec{\eta})
$$

$$
\mathrm{E}_{\mathrm{x}}[\mathrm{F}(\mathrm{x}-[\mathrm{x}]+[\vec{\eta}])]=\int_{\mathrm{C}[0, \mathrm{~T}]} \mathrm{F}(\mathrm{x}-[\mathrm{x}]+[\vec{\eta}]) \mathrm{m}_{\mathrm{w}}(\mathrm{dx}) .
$$

In view of (1.3) and (2.1), we may conclude that

$$
\mathrm{E}\left(\mathrm{F}(\mathrm{x}) \mid \mathrm{X}_{\tau}(\mathrm{x})=\vec{\eta}\right)=\mathrm{E}_{\mathrm{x}}[\mathrm{F}(\mathrm{x}-[\mathrm{x}]+[\vec{\eta}])]
$$

for almost all $\vec{\eta}$ in $\mathbb{R}^{\mathrm{n}}$; i.e., we may express the conditional expectation $\mathrm{E}\left(\mathrm{F} \mid \mathrm{X}_{\tau}\right)(\vec{\eta})$ in terms of an ordinary Wiener integral. Note that for $F \in L_{1}\left(C[0, T], m_{w}\right)$, $\tilde{\mathrm{F}}(\vec{\eta}) \equiv \mathrm{E}\left(\mathrm{F} \mid \mathrm{X}_{\tau}\right)(\vec{\eta})$ is in $\mathrm{L}_{1}\left(\mathbb{R}^{\mathrm{n}}, \mathrm{P}_{\mathrm{X}}(\mathrm{d} \vec{\eta})\right.$. Also note that for each $\mathrm{x} \in \mathrm{C}[0, \mathrm{~T}]$ and each 
partition $\tau=\left\{\mathrm{t}_{1}, \cdots, \mathrm{t}_{\mathrm{n}}\right\}$ of $[0, \mathrm{~T}], \tilde{\mathrm{F}}(\mathrm{x}(\tau))=\mathrm{E}\left(\mathrm{F}(\mathrm{y}) \mid \mathrm{X}_{\tau}(\mathrm{y})=\mathrm{x}(\tau)\right)$ is a function of $\mathrm{x}\left(\mathrm{t}_{1}\right), \cdots, \mathrm{x}\left(\mathrm{t}_{\mathrm{n}}\right)$.

DEFINITION 1. Let $\mathrm{F} \in \mathrm{L}_{1}\left(\mathrm{C}[0, \mathrm{~T}], \mathrm{m}_{\mathrm{w}}\right)$. For each partition $\tau=\left\{\mathrm{t}_{1}, \cdots, \mathrm{t}_{\mathrm{n}}\right\}$ of $[0, T]$ define the operator $\mathscr{D}_{\mathrm{X}(\tau)}$ by

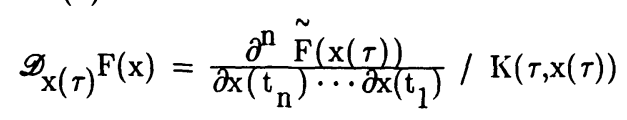

if it exists. Furthermore, if $\mathscr{D}_{\mathrm{x}(\tau)} \mathrm{F}(\mathrm{x})$ exists for each partition $\tau$, then the Wiener derivative of $\mathrm{F}(\mathrm{x})$ is defined by

if the limit exists.

$$
\mathscr{D}_{\mathrm{x}} \mathrm{F}(\mathrm{x})=\lim _{\|\tau\| \rightarrow 0} \mathscr{D}_{\mathrm{x}(\tau)} \mathrm{F}(\mathrm{x})
$$

Our first theorem is the first half of the fundamental theorem of Wiener calculus.

THEOREM 1. Let $F \in L_{1}\left(C[0, T], m_{w}\right)$. Then

$$
\mathscr{D}_{\mathrm{x}} \int_{\mathrm{y} \leq \mathrm{x}} \mathrm{F}(\mathrm{y}) \mathrm{m}_{\mathrm{w}}(\mathrm{dy})=\mathrm{F}(\mathrm{x})
$$

for almost all $x \in C[0, T]$.

PROOF. For $x \in C[0, T]$ let $G(x)$ denote the indefinite Wiener integral

$$
G(x)=\int_{y \leq x} F(y) m_{w}(d y)=E_{y}\left[I_{x}(y) F(y)\right]
$$

where $I_{x}(y)$ is the indicator function

$$
I_{x}(y)= \begin{cases}1 & , \quad y(t) \leq x(t) \text { for all } t \in[0, T] \\ 0, & \text { otherwise. }\end{cases}
$$

Then using (1.7), (2.4), (2.2), (1.3), (2.2) and the Fubini theorem, we obtain

$$
\begin{aligned}
\tilde{\mathrm{G}}(\vec{\eta}) & =\mathrm{E}\left(\mathrm{G}(\mathrm{u}) \mid \mathrm{X}_{\tau}(\mathrm{u})=\vec{\eta}\right) \\
& =\mathrm{E}_{\mathrm{u}}\left(\mathrm{E}_{\mathrm{y}}\left[\mathrm{I}_{\mathrm{u}}(\mathrm{y}) \mathrm{F}(\mathrm{y})\right] \mid \mathrm{X}_{\tau}(\mathrm{u})=\vec{\eta}\right) \\
& =\mathrm{E}_{\mathrm{u}}\left[\mathrm{E}_{\mathrm{y}}\left[\mathrm{I}_{\mathrm{u}-[\mathrm{u}]+[\vec{\eta}]}(\mathrm{y}) \mathrm{F}(\mathrm{y})\right]\right] \\
& =\mathrm{E}_{\mathrm{u}}\left[\int_{\mathbb{R}^{\mathrm{n}}} \mathrm{E}_{\mathrm{y}}\left(\mathrm{I}_{\mathrm{u}-[\mathrm{u}]+[\vec{\eta}]}(\mathrm{y}) \mathrm{F}(\mathrm{y}) \mid \mathrm{X}_{\tau}(\mathrm{y})=\vec{\xi}\right) \mathrm{P}_{\mathrm{X}_{\tau}}(\mathrm{d} \vec{\xi})\right] \\
& =\int_{\mathbb{R}^{\mathrm{n}}} \mathrm{E}_{\mathrm{u}}\left[\mathrm{E}_{\mathrm{y}}\left[\mathrm{I}_{\mathrm{u}-[\mathrm{u}]+[\vec{\eta}]}(\mathrm{y}-[\mathrm{y}]+[\vec{\xi}]) \mathrm{F}(\mathrm{y}-[\mathrm{y}]+[\vec{\xi}])\right]\right] \mathrm{P}_{\mathrm{X}_{\tau}}(\mathrm{d} \vec{\xi})
\end{aligned}
$$

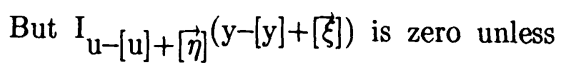

$$
\mathrm{y}(\mathrm{t})-[\mathrm{y}](\mathrm{t})+[\vec{\xi}](\mathrm{t}) \leq \mathrm{u}(\mathrm{t})-[\mathrm{u}](\mathrm{t})+[\vec{\eta}](\mathrm{t})
$$

for all $t \in[0, T]$. But (2.6) implies that

$$
\xi_{j}=y\left(t_{j}\right)-[y]\left(t_{j}\right)+[\xi]\left(t_{j}\right) \leq u\left(t_{j}\right)-[u]\left(t_{j}\right)+[\vec{\eta}]\left(t_{j}\right)=\eta_{j}
$$

for $\mathrm{j}=1, \cdots, \mathrm{n}$. Hence we can write 


$$
\begin{aligned}
\tilde{\mathrm{G}}(\vec{\eta})=\int_{-\infty}^{\eta_{\mathrm{n}}} \cdots \int_{-\infty}^{\eta_{1}} \mathrm{E}_{\mathrm{u}}\left[\mathrm { E } _ { \mathrm { y } } \left[\mathrm{I}_{\mathrm{u}-[\mathrm{u}]+[\vec{\eta}]}(\mathrm{y}-[\mathrm{y}]+[\vec{\xi}])\right.\right. \\
\cdot \mathrm{F}(\mathrm{y}-[\mathrm{y}]+[\vec{\xi}])]] \mathrm{K}(\tau, \vec{\xi}) \mathrm{d} \xi_{1} \cdots \mathrm{d} \xi_{\mathrm{n}},
\end{aligned}
$$

and so for each $x \in C[0, T]$,

$$
\begin{aligned}
\tilde{G}(x(\tau))= & \int_{-\infty}^{x\left(t_{n}\right)} \cdots \int_{-\infty}^{x\left(t_{1}\right)} E_{u}\left[E _ { y } \left[I_{u-[u]}[x(\tau)]\right.\right. \\
& \left.F(y-[y]+[\xi])]] K(\tau, \xi) d \xi_{1} \cdots d \xi_{n} .[\xi]\right)
\end{aligned}
$$

Hence

$$
\begin{aligned}
\frac{\partial^{\mathrm{n}} \tilde{\mathrm{G}}(\mathrm{x}(\tau))}{\partial \mathrm{x}\left(\mathrm{t}_{\mathrm{n}}\right) \cdot \cdots \partial \mathrm{x}\left(\mathrm{t}_{1}\right)}= & \mathrm{E}_{\mathrm{u}}\left[\mathrm { E } _ { \mathrm { y } } \left[\mathrm{I}_{\mathrm{u}-[\mathrm{u}]+[\mathrm{x}(\tau)]}(\mathrm{y}-[\mathrm{y}]+[\mathrm{x}(\mathrm{t})])\right.\right. \\
& \cdot \mathrm{F}(\mathrm{y}-[\mathrm{y}]+[\mathrm{x}(\tau)])]] \mathrm{K}(\tau, \mathrm{x}(\tau)) \\
= & \mathrm{E}_{\mathrm{u}, \mathrm{y}}\left(\mathrm{I}_{\mathrm{u}}(\mathrm{y}) \mathrm{F}(\mathrm{y}) \mid \mathrm{X}_{\tau}(\mathrm{y})=\mathrm{x}(\tau), \mathrm{X}_{\tau}(\mathrm{u})=\mathrm{x}(\tau)\right) \mathrm{K}(\tau, \mathrm{x}(\tau))
\end{aligned}
$$

Applying (1.9) to (2.7) yields

$$
\begin{aligned}
\mathscr{D}_{\mathrm{X}}(\mathrm{G}(\mathrm{x})) & =\lim _{\|\tau\| \rightarrow 0} \mathrm{E}_{\mathrm{u}, \mathrm{y}}\left(\mathrm{I}_{\mathrm{u}}(\mathrm{y}) \mathrm{F}(\mathrm{y}) \mid \mathrm{X}_{\tau}(\mathrm{y})=\mathrm{x}(\tau), \mathrm{X}_{\tau}(\mathrm{u})=\mathrm{x}(\tau)\right) \\
& =\mathrm{F}(\mathrm{x})
\end{aligned}
$$

for almost all $x$ in $C[0, T]$ which concludes the proof of Theorem 1 .

COROLlaRY 1. If $\left\{\mathrm{t}_{1}^{\prime}, \cdots, \mathrm{t}_{\mathrm{m}}^{\prime}\right\} \subseteq \tau=\left\{\mathrm{t}_{1}, \cdots, \mathrm{t}_{\mathrm{n}}\right\}$ and if $\mathrm{F}(\mathrm{y})=\mathrm{f}\left(\mathrm{y}\left(\mathrm{t}_{1}^{\prime}\right), \cdots, \mathrm{y}^{\prime}\left(\mathrm{t}_{\mathrm{m}}^{\prime}\right)\right)$ is in $\mathrm{L}_{1}\left(\mathrm{C}[0, \mathrm{~T}], \mathrm{m}_{\mathrm{w}}\right)$, then

$$
\mathscr{D}_{\mathrm{x}(\tau)} \int_{\mathrm{y} \leq \mathrm{x}} \mathrm{F}(\mathrm{y}) \mathrm{m}_{\mathrm{w}}(\mathrm{dy})=\mathrm{F}(\mathrm{x}) \mathrm{E}_{\mathrm{u}, \mathrm{y}}\left(\mathrm{I}_{\mathrm{u}}(\mathrm{y}) \mid \mathrm{X}_{\tau}(\mathrm{y})=\mathrm{X}_{\tau}(\mathrm{u})=\mathrm{x}(\tau)\right)
$$

and

$$
\mathscr{D}_{\mathrm{x}} \int_{\mathrm{y} \leq \mathrm{x}} \mathrm{F}(\mathrm{y}) \mathrm{m}_{\mathrm{w}}(\mathrm{dy})=\mathrm{F}(\mathrm{x})=\mathrm{f}\left(\mathrm{x}\left(\mathrm{t}_{1}^{\prime}\right), \cdots, \mathrm{x}\left(\mathrm{t}_{\mathrm{n}}^{\prime}\right)\right)
$$

for almost all $\mathrm{x}$ in $\mathrm{C}[0, \mathrm{~T}]$.

PROOF. Using (2.7) and (2.4) we see that

$$
\mathscr{D}_{\mathrm{X}(\tau)} \int_{\mathrm{y} \leq \mathrm{x}} \mathrm{F}(\mathrm{y}) \mathrm{m}_{\mathrm{w}}(\mathrm{dy})=\mathrm{E}_{\mathrm{u}, \mathrm{y}}\left(\mathrm{I}_{\mathrm{u}}(\mathrm{y}) \mathrm{F}(\mathrm{y}) \mid \mathrm{X}_{\tau}(\mathrm{y})=\mathrm{x}(\tau), \mathrm{X}_{\tau}(\mathrm{u})=\mathrm{x}(\tau)\right) .
$$

Under the conditioning $\mathrm{X}_{\tau}(\mathrm{y})=\mathrm{x}(\tau), \mathrm{F}(\mathrm{y})$ becomes $\mathrm{f}\left(\mathrm{x}\left(\mathrm{t}_{1}^{\prime}\right), \cdots, \mathrm{x}\left(\mathrm{t}_{\mathrm{m}}^{\prime}\right)\right)$ which equals $\mathrm{F}(\mathrm{x})$. Therefore,

$$
\mathscr{D}_{\mathrm{x}(\tau)} \int_{\mathrm{y} \leq \mathrm{x}} \mathrm{F}(\mathrm{y}) \mathrm{m}_{\mathrm{w}}(\mathrm{dy})=\mathrm{F}(\mathrm{x}) \mathrm{E}_{\mathrm{u}, \mathrm{y}}\left(\mathrm{I}_{\mathrm{u}}(\mathrm{y}) \mid \mathrm{X}_{\tau}(\mathrm{y})=\mathrm{X}_{\tau}(\mathrm{u})=\mathrm{x}(\tau)\right) .
$$

As $\|\tau\| \rightarrow 0, \mathrm{E}_{\mathrm{u}, \mathrm{y}}\left(\mathrm{I}_{\mathrm{u}}(\mathrm{y}) \mid \mathrm{X}_{\tau}(\mathrm{y})=\mathrm{X}_{\tau}(\mathrm{u})=\mathrm{x}(\tau)\right) \rightarrow \mathrm{I}_{\mathrm{x}}(\mathrm{x})=1$ by (1.9) for almost all $\mathrm{x}$ in $\mathrm{C}[0, \mathrm{~T}]$. Thus Corollary 1 is established.

COROLLARY 2. Let $\tau^{\prime}=\left\{\mathrm{t}_{1}^{\prime}, \cdots, \mathrm{t}_{\mathrm{m}}^{\prime}\right\}$ be any partition of $[0, \mathrm{~T}]$, and let $\mathrm{F}(\mathrm{x})=\mathrm{f}\left(\mathrm{x}\left(\mathrm{t}_{1}^{\prime}\right), \cdots, \mathrm{x}\left(\mathrm{t}_{\mathrm{m}}^{\prime}\right)\right)$ be in $\mathrm{L}_{1}\left(\mathrm{C}[0, \mathrm{~T}], \mathrm{m}_{\mathrm{w}}\right)$. Then $\mathscr{D}_{\mathrm{x}} \mathrm{F}(\mathrm{x})=0$. 
PROOF. Let $\tau$ be a partition of $[0, T]$ properly containing $\tau^{\prime}$. Then

$$
\left.\tilde{\mathrm{F}}(\mathrm{x}(\tau))=\mathrm{E}(\mathrm{F} \mid \mathrm{y}) \mid \mathrm{X}_{\tau}(\mathrm{y})=\mathrm{x}(\tau)\right)=\mathrm{f}\left(\mathrm{x}^{\prime}\left(\mathrm{t}_{1}^{\prime}\right), \cdots, \mathrm{x}\left(\mathrm{t}_{\mathrm{n}}^{\prime}\right)\right) .
$$

Thus $\mathscr{D}_{\mathrm{x}(\tau)} \mathrm{F}(\mathrm{x})=0$, and so $\mathscr{D}_{\mathrm{X}} \mathrm{F}(\mathrm{x})=0$.

\section{LEBESGUE AND WIENER ABSOLUTE CONTINUITY.}

In this section we show that certain functions defined on $C[0, T]$ are equal to the indefinite integral of their Wiener derivative.

For $\vec{a}=\left(a_{1}, \cdots, a_{n}\right)$ and $\vec{b}=\left(b_{1}, \cdots, b_{n}\right)$ in $\mathbb{R}^{n}$ with $a_{i}<b_{i}, i=1, \cdots, n$, let $\mathrm{V}(\vec{a}, \vec{b}, \mathrm{k})$ be the collection of all points of the form $\vec{v}=\left(v_{1}, \cdots, v_{n}\right)$ where each $v_{i}$ is either $a_{i}$ or $b_{i}$ and exactly $k$ of the $v_{i}$ are $a_{i}$ 's. For any function $f$ defined on $v(\vec{a}, \vec{b}, k)$ for $\mathrm{k}=0,1, \cdots, \mathrm{n}$, let

$$
\Delta_{\vec{a}, \vec{b}} f=f(\vec{b})+\sum_{k=1}^{n}(-1)^{k} \sum_{\vec{v} \in V(\vec{a}, \vec{b}, k)}^{\sum} f(\vec{v}) .
$$

A function of $n$ variables $f\left(u_{1}, \cdots, u_{n}\right)$ is said to be Lebesgue absolutely continuous in the sense of Vitali (see Clarkson and Adams [6,7] and Hobson [8]) on the region $\Omega \subset \mathbb{R}^{\mathrm{n}}$ if,

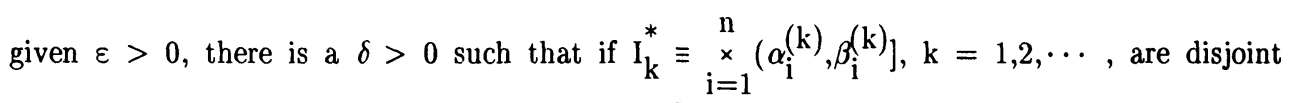
n-dimensional rectangles contained in $\Omega$ with $\underset{\mathrm{k}=1}{\mathrm{~N}} \mathrm{~m}_{\mathrm{L}}\left(\mathrm{I}_{\mathrm{k}}^{*}\right)<\delta$ for any $\mathrm{N}$, then $\sum_{\mathrm{k}=1}^{\mathrm{N}}\left|\Delta_{\vec{\alpha}}(\mathrm{k}), \vec{\beta}^{(\mathrm{k})} \mathrm{f}\right|<\varepsilon$, where $\mathrm{m}_{\mathrm{L}}(\cdot)$ denotes $\mathrm{n}$-dimensional Lebesgue measure, and $\vec{\alpha}^{(\mathrm{k})}=\left(\alpha_{1}^{(\mathrm{k})}, \cdots, \alpha_{\mathrm{n}}^{(\mathrm{k})}\right)$. A function $\mathrm{f}\left(\mathrm{u}_{1}, \cdots, \mathrm{u}_{\mathrm{n}}\right)$ is said to be Lebesgue absolutely continuous (in the sense of Hardy-Krause; see Berkson and Gillespie [9], and Clarkson and Adams $[6,7])$ on a region $\Omega \subset \mathbb{R}^{\mathrm{n}}$ if for each $\mathrm{k}=1, \cdots, \mathrm{n}-1$, whenever $\mathrm{n}-\mathrm{k}$ variables are fixed then $f$, as a function of its remaining $k$ variables, is Lebesgue absolutely continuous in the sense of Vitali on $\Omega \cap \mathbb{R}^{\mathrm{k}}$. When we merely state "Lebesgue absolutely continuous", it is always meant in the sense of Hardy-Krause.

It is well known that if $f\left(u_{1}, \cdots, u_{n}\right)$ is Lebesgue absolutely continuous in the sense of Vitali on $\mathrm{R} \equiv \underset{\mathrm{i}=1}{\mathrm{n}}\left[\mathrm{a}_{\mathrm{i}}, \mathrm{b}_{\mathrm{i}}\right]$, then $\partial^{\mathrm{n}_{\mathrm{f}}}\left(\mathrm{u}_{1}, \cdots, \mathrm{u}_{\mathrm{n}}\right) / \partial \mathrm{u}_{1} \cdots \partial \mathrm{u}_{\mathrm{n}}$ exists a.e. on $\mathrm{R}$ and is integrable on R. Furthermore

$$
\int_{R}\left[\partial^{n_{f}}\left(u_{1}, \cdots, u_{n}\right) / \partial u_{1} \cdots \partial u_{n}\right] d u_{1} \cdots d u_{n}=\Delta_{\vec{a}, b} f
$$

and

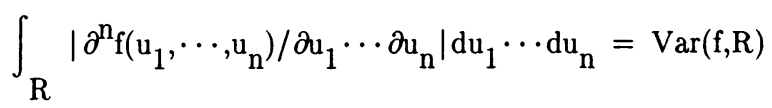

where $\operatorname{Var}(f, R)$ denotes the total variation of $f$ over $R$. 
Let $\mathrm{G}(\mathrm{x})$ be any Wiener integrable function on $\mathrm{C}[0, \mathrm{~T}]$. Then, by definition, $\tilde{\mathrm{G}}(\vec{\eta})=\mathrm{E}\left(\mathrm{G} \mid \mathrm{X}_{\tau}\right)(\vec{\eta})$ is a function of $\vec{\eta}$ which is integrable with respect to $\mathrm{P}_{\mathrm{X}}(\mathrm{d} \vec{\eta})=\mathrm{K}(\tau, \vec{\eta}) \mathrm{d} \vec{\eta}$.

DEFINITION 2. A Wiener integrable function $\mathrm{G}(\mathrm{x})$ defined on $\mathrm{C}[0, \mathrm{~T}]$ is said to be Wiener absolutely continuous provided that for each $\varepsilon>0$ there exists a $\delta>0$ such that if the sequence $\mathrm{I}_{\mathrm{k}} \equiv\left\{\mathrm{x} \in \mathrm{C}[0, \mathrm{~T}]: \alpha_{\mathrm{i}}^{(\mathrm{k})}<\mathrm{x}\left(\mathrm{s}_{\mathrm{i}}^{(\mathrm{k})}\right) \leq \beta_{\mathrm{i}}^{(\mathrm{k})}, \mathrm{i}=1, \cdots, \mathrm{m}^{(\mathrm{k})}\right\}$ with $-\infty \leq \alpha_{\mathrm{i}}^{(\mathrm{k})}<\beta_{\mathrm{i}}^{(\mathrm{k})} \leq \infty$ are disjoint Wiener intervals with $\underset{\mathrm{k}=1}{\mathrm{~N}} \mathrm{~m}_{\mathrm{w}}\left(\mathrm{I}_{\mathrm{k}}\right)<\delta$ for any $\mathrm{N}$, then $\sum_{\mathrm{k}=1}^{\mathrm{N}}\left|\Delta_{\vec{\alpha}^{(\mathrm{k})}, \vec{\beta}^{(\mathrm{k})}} \tilde{\mathrm{G}}\right|<\varepsilon$.

The following propositions can be easily established.

PROPOSITION A. If $\mathrm{G}(\mathrm{x})$ is Wiener absolutely continuous on $\mathrm{C}[0, \mathrm{~T}]$, then for every partition $\tau$ of $(0, T], \tilde{G}(\vec{\eta})$ is Lebesgue absolutely continuous on $\mathbb{R}^{|\tau|}$, where $|\tau|$ denotes the number of points in $\tau$.

PROPOSITION B. Let $\mathrm{F} \in \mathrm{L}_{1}\left(\mathrm{C}[0, \mathrm{~T}], \mathrm{m}_{\mathrm{w}}\right)$. Then the indefinite Wiener integral $G(x)=\int_{y \leq x} F(y) m_{w}(d y)$ is Wiener absolutely continuous on $C[0, T]$.

Our next theorem is the second half of the fundamental theorem of Wiener Calculus.

THEOREM 2. Let $\mathrm{G} \in \mathrm{L}_{1}\left(\mathrm{C}[0, \mathrm{~T}], \mathrm{m}_{\mathrm{w}}\right)$ satisfy the conditions:

(i) $\mathscr{D}_{\mathrm{x}} \mathrm{G}(\mathrm{x})$ exists for almost all $\mathrm{x} \in \mathrm{C}[0, \mathrm{~T}]$ and belongs to $\mathrm{L}_{1}\left(\mathrm{C}[0, \mathrm{~T}], \mathrm{m}_{\mathrm{w}}\right)$,

(ii) $\mathrm{G}(\mathrm{x})$ is Wiener absolutely continuous on $\mathrm{C}[0, \mathrm{~T}]$,

(iii) If $\left\{\mathrm{x}_{\mathrm{k}}\right\}_{\mathrm{k}=1}^{\infty}$ is a sequence in $\mathrm{C}[0, \mathrm{~T}]$ such that $\mathrm{x}_{\mathrm{k}}\left(\mathrm{s}_{0}\right) \rightarrow-\infty$ as $\mathrm{k} \rightarrow \infty$ for some fixed point $\mathrm{s}_{0} \in(0, \mathrm{~T}]$, then $\mathrm{G}\left(\mathrm{x}_{\mathrm{k}}\right) \rightarrow 0$ as $\mathrm{k} \rightarrow \infty$.

Then

$$
\mathrm{G}(\mathrm{u})=\int_{\mathrm{x} \leq \mathrm{u}} \mathscr{D}_{\mathrm{x}} \mathrm{G}(\mathrm{x}) \mathrm{m}_{\mathrm{w}}(\mathrm{dx})
$$

for almost all $\mathrm{u}$ in $\mathrm{C}[0, \mathrm{~T}]$.

PROOF. For given $\varepsilon>0$ let $\delta=\delta(\varepsilon / 3)>0$ be the value for the Wiener absolute continuity of $\mathrm{G}(\mathrm{x})$, and also assume that

$$
\mathrm{m}_{\mathrm{w}}(\mathrm{S})<\delta \Rightarrow \int_{\mathrm{S}}\left|\mathscr{D}_{\mathrm{x}} \mathrm{G}(\mathrm{x})\right| \mathrm{m}_{\mathrm{w}}(\mathrm{dx})<\varepsilon / 3 .
$$

Let $\left\{\tau^{(\mathrm{k})}\right\}$ be a sequence of partitions of $[0, \mathrm{~T}]$ such that $\left\|\tau^{(\mathrm{k})}\right\| \rightarrow 0$ as $\mathrm{k} \rightarrow \infty$. Then

$$
\lim _{\mathrm{k} \rightarrow \infty} \mathscr{D}_{\mathrm{x}\left(\tau^{(\mathrm{k})}\right)} \mathrm{G}(\mathrm{x})=\mathscr{D}_{\mathrm{x}} \mathrm{G}(\mathrm{x}) \text { for almost all } \mathrm{x} \in \mathrm{C}[0, \mathrm{~T}] \text {. }
$$

By Egoroff's theorem, there exists a set $\mathrm{C}_{\varepsilon} \subset \mathrm{C}[0, \mathrm{~T}]$ with $\mathrm{m}_{\mathrm{w}}\left(\mathrm{C}_{\varepsilon}\right)>1-\delta / 2$ and a positive integer $\mathrm{k}_{0}$ such that if $\mathrm{k} \geq \mathrm{k}_{0}$, then 


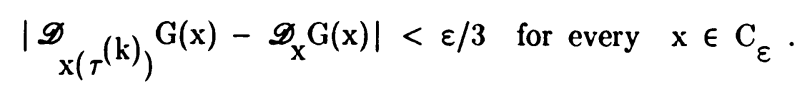

Let

$$
\mathrm{C}_{\mathrm{k}}=\left\{\mathrm{x} \in \mathrm{C}[0, \mathrm{~T}]:\left|\mathscr{D}_{\mathrm{x}\left(\tau^{(\mathrm{k})}\right)} \mathrm{G}(\mathrm{x})-\mathscr{D}_{\mathrm{X}} \mathrm{G}(\mathrm{x})\right|<\varepsilon / 3\right\}, \mathrm{k} \geq \mathrm{k}_{0} .
$$

Then $\mathrm{C}_{\varepsilon} \subset \mathrm{C}_{\mathrm{k}}$, and hence $\mathrm{m}_{\mathrm{w}}\left(\mathrm{C}_{\mathrm{k}}\right) \geq \mathrm{m}_{\mathrm{w}}\left(\mathrm{C}_{\varepsilon}\right)>1-\delta / 2$, and

$$
\int_{\mathrm{C}_{\mathrm{k}}}\left|\mathscr{D}_{\mathrm{x}\left(\tau^{(\mathrm{k})}\right)} \mathrm{G}(\mathrm{x})-\mathscr{D}_{\mathrm{x}} \mathrm{G}(\mathrm{x})\right| \mathrm{m}_{\mathrm{w}}(\mathrm{dx})<\varepsilon / 3 \text { for } \mathrm{k} \geq \mathrm{k}_{0} \text {. }
$$

The complements satisfy $\mathrm{m}_{\mathrm{w}}\left(\mathrm{C}_{\mathrm{k}}^{\sim}\right) \leq \mathrm{m}_{\mathrm{w}}\left(\mathrm{C}_{\varepsilon}^{\sim}\right)<\delta / 2$ for $\mathrm{k} \geq \mathrm{k}_{0}$. Next consider fixed $\mathrm{k}$, $\mathrm{k} \geq \mathrm{k}_{0}$ and let $\mathrm{q}$ denote the number of points in the partition $\tau^{(\mathrm{k})}$. Let

$$
\mathrm{E}_{\mathrm{k}} \equiv\left\{\vec{\eta}=\left(\eta_{1}, \cdots, \eta_{\mathrm{q}}\right) \in \mathbb{R}^{\mathrm{q}}: \vec{\eta}=\mathrm{x}\left(\tau^{(\mathrm{k})}\right) \text { for some } \mathrm{x} \in \mathrm{C}_{\mathrm{k}}^{\sim}\right\} \text {. }
$$

Then,

$$
\mathrm{m}_{\mathrm{w}}\left(\mathrm{C}_{\mathrm{k}}^{\sim}\right)=\int_{\mathrm{E}_{\mathrm{k}}} \mathrm{K}\left(\tau^{(\mathrm{k})}, \vec{\eta}\right) \mathrm{d} \vec{\eta}
$$

Since $\mathrm{K}\left(\tau^{(\mathrm{k})}, \vec{\eta}\right)$ is bounded in $\vec{\eta}$ on $\mathbb{R}^{\mathrm{q}}$ and $\int_{\mathrm{E}_{\mathrm{k}}} \mathrm{K}\left(\tau^{(\mathrm{k})}, \vec{\eta}\right) \mathrm{d} \vec{\eta}=\mathrm{m}_{\mathrm{w}}\left(\mathrm{C}_{\mathrm{k}}^{\sim}\right)<\delta / 2$, we can find a countable sequence of disjoint $q$-dimensional rectangles $\mathrm{I}_{\ell}^{*}=\underset{\mathrm{i}=1}{\mathrm{q}}\left(\alpha_{\mathrm{i}}^{(\ell)}, \beta_{\mathrm{i}}^{(\ell)}\right], \ell=1,2, \cdots$ such that $\mathrm{E}_{\mathrm{k}} \subset \bigcup_{\ell=1}^{\infty} \mathrm{I}_{\ell}^{*}$, and

$$
\int_{\mathrm{E}_{\mathrm{k}}} \mathrm{K}\left(\tau^{(\mathrm{k})}, \vec{\eta}\right) \mathrm{d} \vec{\eta} \leq \sum_{\ell=1}^{\infty} \int_{\mathrm{I}_{\ell}} \mathrm{K}\left(\tau^{(\mathrm{k})}, \vec{\eta}\right) \mathrm{d} \vec{\eta}=\sum_{\ell=1}^{\infty} \mathrm{m}_{\mathrm{w}}(\mathrm{I} \ell)<\delta
$$

where

$$
\mathrm{I}_{\ell}=\left\{\mathrm{x} \in \mathrm{C}[0, \mathrm{~T}]: \alpha_{\mathrm{i}}^{(\ell)}<\mathrm{x}\left(\mathrm{s}_{\mathrm{i}}^{(\mathrm{k})}\right) \leq \beta_{\mathrm{i}}^{(\ell)} \text { for each } \mathrm{s}_{\mathrm{i}}^{(\mathrm{k})} \in \tau^{(\mathrm{k})}\right\}
$$

Hence

$$
\begin{aligned}
& \int_{\mathrm{C}_{\mathrm{k}}^{\sim}}\left|\mathscr{D} \mathrm{x}\left(\tau^{(\mathrm{k})}\right) \mathrm{G}(\mathrm{x})\right| \mathrm{m}_{\mathrm{w}}(\mathrm{dx})=\int_{\mathrm{E}_{\mathrm{k}}}\left|\partial^{\mathrm{q}} \tilde{\mathrm{G}}\left(\eta_{1}, \cdots, \eta_{\mathrm{q}}\right) / \partial \eta_{1} \cdots \partial \eta_{\mathrm{q}}\right| \mathrm{d} \eta_{1} \cdots \mathrm{d} \eta_{\mathrm{q}} \\
& \leq \sum_{\ell=1}^{\infty} \int_{\alpha_{\mathrm{q}}^{(\ell)}}^{\beta_{\mathrm{q}}^{(\ell)}} \cdots \int_{\alpha_{1}^{(\ell)}}^{\beta_{1}^{(\ell)}}\left|\partial^{\mathrm{q}} \tilde{\mathrm{G}}\left(\eta_{1}, \cdots, \eta_{\mathrm{q}}\right) / \partial \eta_{1} \cdots \partial \eta_{\mathrm{q}}\right| \mathrm{d} \eta_{1} \cdots \mathrm{d} \eta_{\mathrm{q}} \\
& =\sum_{\ell=1}^{\infty} \operatorname{Var}\left({\tilde{\mathrm{G}}, \mathrm{I}_{\ell}^{*}}^{*}\right),
\end{aligned}
$$

where the last equality follows from (3.3). Now,

$$
\operatorname{Var}\left(\tilde{\mathrm{G}}, \mathrm{I}_{\ell}^{*}\right)=\sup \sum_{\mathrm{i}}\left|\Delta_{\vec{\sigma}_{\mathrm{i}}, \vec{\rho}_{\mathrm{i}}} \underset{\mathrm{G}}{\tilde{\eta}}(\vec{\eta})\right|
$$

where the supremum is taken over all possible nets of $\mathrm{I}_{\ell}^{*}$, and each net has total Lebesgue 
measure equal to that of $\mathrm{I}_{\ell}^{*}$, and so the corresponding Wiener intervals have total measure equal to $\mathrm{m}_{\mathrm{w}}\left(\mathrm{I}_{\ell}\right)$. Since $\sum_{\ell=1}^{\infty} \mathrm{m}_{\mathrm{w}}\left(\mathrm{I}_{\ell}\right)<\delta(\varepsilon / 3)$, by the Wiener absolute continuity of $\mathrm{G}$, we have

$$
\sum_{\ell=1}^{\mathrm{N}} \sum_{\mathrm{i}}\left|\Delta_{\vec{\sigma}_{\mathrm{i}}, \vec{\rho}_{\mathrm{i}}} \tilde{\mathrm{G}}(\vec{\eta})\right|<\varepsilon / 3 \text { for every } \mathrm{N} \text { and every net. }
$$

Thus, by taking the supremum over all nets, we get

$$
\sum_{\ell=1}^{N} \operatorname{Var}\left(\tilde{G}^{*} I_{\ell}^{*}\right) \leq \varepsilon / 3 \text { for every } \mathrm{N},
$$

and hence

$$
\int_{\mathrm{C}_{\mathrm{k}}^{\sim}}\left|\mathscr{D} \mathrm{x}\left(\tau^{(\mathrm{k})}\right) \mathrm{G}(\mathrm{x})\right| \mathrm{m}_{\mathrm{w}}(\mathrm{dx}) \leq \varepsilon / 3
$$

Thus, for every $\mathrm{k} \geq \mathrm{k}_{0}$,

$$
\begin{aligned}
& \int_{\mathrm{C}[0, \mathrm{~T}]}\left|\mathscr{D}_{\mathrm{x}\left(\tau^{(\mathrm{k})}\right)} \mathrm{G}(\mathrm{x})-\mathscr{D}_{\mathrm{x}} \mathrm{G}(\mathrm{x})\right| \mathrm{m}_{\mathrm{w}}(\mathrm{dx}) \\
& \leq \int_{\mathrm{C}_{\mathrm{k}}}\left|\mathscr{D}\left(\tau^{(\mathrm{k})}\right) \mathrm{G}(\mathrm{x})-\mathscr{D}_{\mathrm{x}} \mathrm{G}(\mathrm{x})\right| \mathrm{m}_{\mathrm{w}}(\mathrm{dx}) \\
& \quad+\int_{\mathrm{C}_{\mathrm{k}}^{\sim}}\left|\mathscr{D}\left(\tau^{(\mathrm{k})}\right) \mathrm{G}(\mathrm{x})\right| \mathrm{m}_{\mathrm{w}}(\mathrm{dx})+\int_{\mathrm{C}_{\mathrm{k}}^{\sim}}\left|\mathscr{D}_{\mathrm{x}} \mathrm{G}(\mathrm{x})\right| \mathrm{m}_{\mathrm{w}}(\mathrm{dx}) \\
& <\varepsilon .
\end{aligned}
$$

In particular

$$
\int_{[\mathrm{x}] \leq[\mathrm{u}]} \mid \mathscr{D} \mathrm{x}\left(\tau(\mathrm{k})^{(\mathrm{k})} \mathrm{G}(\mathrm{x})-\mathscr{D}_{\mathrm{x}} \mathrm{G}(\mathrm{x}) \mid \mathrm{m}_{\mathrm{w}}(\mathrm{dx})<\varepsilon \text { for } \mathrm{k} \geq \mathrm{k}_{0}\right.
$$

where $[\cdot]$ corresponds to $\tau^{(\mathrm{k})}$. Hence

$$
\lim _{\mathrm{k} \rightarrow \infty}\left[\int_{[\mathrm{x}] \leq[\mathrm{u}]} \mathscr{D}_{\mathrm{x}\left(\tau^{(\mathrm{k})}\right)} \mathrm{G}(\mathrm{x}) \mathrm{m}_{\mathrm{w}}(\mathrm{dx})-\int_{[\mathrm{x}] \leq[\mathrm{u}]} \mathscr{D}_{\mathrm{x}} \mathrm{G}(\mathrm{x}) \mathrm{m}_{\mathrm{w}}(\mathrm{dx})\right]=0 .
$$

Since $\{x \in C[0, T]:[x] \leq[u]\} \rightarrow\{x \in C[0, T]: x \leq u\}$ as $k \rightarrow \infty$, an application of the dominated convergence theorem yields

Thus,

$$
\lim _{\mathrm{k} \rightarrow \infty} \int_{[\mathrm{x}] \leq[\mathrm{u}]} \mathscr{D}_{\mathrm{x}} \mathrm{G}(\mathrm{x}) \mathrm{m}_{\mathrm{w}}(\mathrm{dx})=\int_{\mathrm{x} \leq \mathrm{u}} \mathscr{D}_{\mathbf{x}} \mathrm{G}(\mathrm{x}) \mathrm{m}_{\mathrm{w}}(\mathrm{dx}) .
$$

$$
\lim _{\mathrm{k} \rightarrow \infty} \int_{[\mathrm{x}] \leq[\mathrm{u}]} \mathscr{D}_{\mathrm{x}\left(\tau^{(\mathrm{k})}\right)} \mathrm{G}(\mathrm{x}) \mathrm{m}_{\mathrm{w}}(\mathrm{dx})=\int_{\mathrm{x} \leq \mathrm{u}} \mathscr{D}_{\mathrm{x}} \mathrm{G}(\mathrm{x}) \mathrm{m}_{\mathrm{w}}(\mathrm{dx}) .
$$

On the other hand, using (2.3), (3.2) and (3.1), we see that for any a $\in C[0, T]$ with $\mathrm{a}<\mathrm{u}$, 


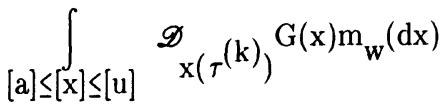

$$
\begin{aligned}
& =\int_{\mathrm{a}\left(\tau^{(\mathrm{k})}\right) \leq \mathrm{x}\left(\tau^{(\mathrm{k})}\right) \leq \mathrm{u}\left(\tau^{(\mathrm{k})}\right)}{ }_{\mathrm{x}\left(\tau^{(\mathrm{k})}\right)}^{\mathrm{G}(\mathrm{x}) \mathrm{m}_{\mathrm{w}}(\mathrm{dx})} \\
& =\int_{\mathrm{a}\left(\mathrm{s}_{\mathrm{q}}^{(\mathrm{k})}\right)}^{\mathrm{u}\left(\mathrm{s}_{\mathrm{q}}^{(\mathrm{k})}\right)} \cdots \int_{\mathrm{a}\left(\mathrm{s}_{1}^{(\mathrm{k})}\right)}^{\mathrm{u}\left(\mathrm{s}_{1}^{(\mathrm{k})}\right)}\left[\partial^{\mathrm{q}} \tilde{\mathrm{G}}\left(\eta_{1}, \cdots, \eta_{\mathrm{q}}\right) / \partial \eta_{1} \cdots \partial \eta_{\mathrm{q}}\right] \mathrm{d} \eta_{1} \cdots \mathrm{d} \eta_{\mathrm{q}} \\
& =\Delta_{\mathrm{a}\left(\tau^{(\mathrm{k})}\right), \mathrm{u}\left(\tau^{(\mathrm{k})}\right)} \tilde{\mathrm{G}} \\
& =\tilde{\mathrm{G}}\left(\mathrm{u}\left(\tau^{(\mathrm{k})}\right)\right)+\sum_{\ell=1}^{\mathrm{q}}(-1)^{\ell} \sum_{\overrightarrow{\mathrm{v}} \in \mathrm{V}\left(\mathrm{a}\left(\tau^{(\mathrm{k})}\right), \mathrm{u}\left(\tau^{(\mathrm{k})}\right), \ell\right)} \tilde{\mathrm{G}}(\overrightarrow{\mathrm{v}}) .
\end{aligned}
$$

If we let $\mathrm{a}\left(\mathrm{s}_{\mathrm{i}}^{(\mathrm{k})}\right) \rightarrow-\infty$ as $\mathrm{k} \rightarrow \infty$ for $\mathrm{i}=1, \cdots, \mathrm{q}$ in $(3.6)$, then by assumption (iii), $\tilde{\mathrm{G}}(\overrightarrow{\mathrm{v}}) \rightarrow 0$ as $\mathrm{k} \rightarrow \infty$ for every $\overrightarrow{\mathrm{v}} \in \mathrm{V}\left(\mathrm{a}\left(\tau^{(\mathrm{k})}\right), \mathrm{u}\left(\tau^{(\mathrm{k})}\right), \ell\right), \ell \geq 1$. Thus (3.6) reduces to

$$
\int_{[\mathrm{x}] \leq[\mathrm{u}]} \mathscr{D} \mathrm{x}\left(\tau^{(\mathrm{k})}\right) \mathrm{G}(\mathrm{x}) \mathrm{m}_{\mathrm{w}}(\mathrm{dx})=\tilde{\mathrm{G}}\left(\mathrm{u}\left(\tau^{(\mathrm{k})}\right)\right) .
$$

In view of (1.9) and (3.5), we conclude that

$$
\int_{\mathrm{x} \leq \mathrm{u}} \mathscr{D}_{\mathrm{x}} \mathrm{G}(\mathrm{x}) \mathrm{m}_{\mathrm{w}}(\mathrm{dx})=\mathrm{G}(\mathrm{u}) \text { for almost all } \mathrm{u} \text { in } \mathrm{C}[0, \mathrm{~T}] \text {, }
$$

and so (3.4) is established.

\section{REFERENCES}

1. DOOB, J.L. Stochastic Processes, Wiley and Sons, 1965.

2. TUCKER, H.G. A Graduate Course in Probability, Academic Press, 1967.

3. YEH, J. Inversion of Conditional Expectation, Pacif. J. Math. $\underline{52}$ (1974), 631-640.

4. SMOLOWITZ, L.H. Wiener Derivatives and Corresponding Fundamental Theorem of the Integral Calculus, Doctoral thesis, University of Minnesota, (1967).

5. PARK, C. and SKOUG, D.L. A Simple Formula for Conditional Wiener Integrals with Applications, Pacif. J. Math. 135(1988), 381-394.

6. CLARKSON, J.A. and ADAMS, C.R. On Definitions of Bounded Variation for Functions of Two Variables, Tran. Amer. Math. Soc. 35(1933), 824-854.

7. CLARKSON, J.A. and ADAMS, C.R. Properties of Functions $f(x, y)$ of Bounded Variation, Tran. Amer. Math. Soc. 36(1934), 711-730.

8. HOBSON, E.W. The Theory of Functions of a Real Variable and the Theory of Fourier Series, Vol. 1, Cambridge University Press, 1927.

9. BERKSON, E. and GILLESPIE, T.A. Absolutely Continuous Functions of Two Variables and Well Bounded Operators, J. London Math. Soc. 30(1984), 305-321. 


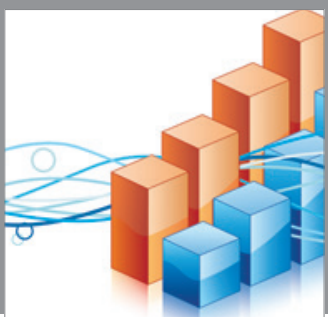

Advances in

Operations Research

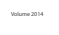

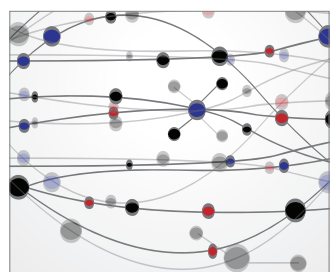

\section{The Scientific} World Journal
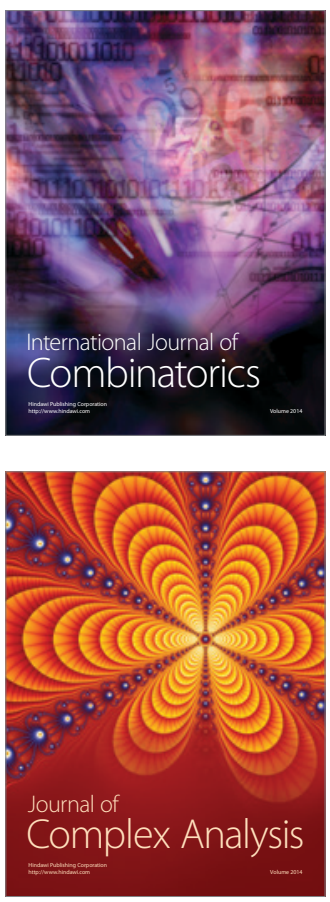

International Journal of

Mathematics and

Mathematical

Sciences
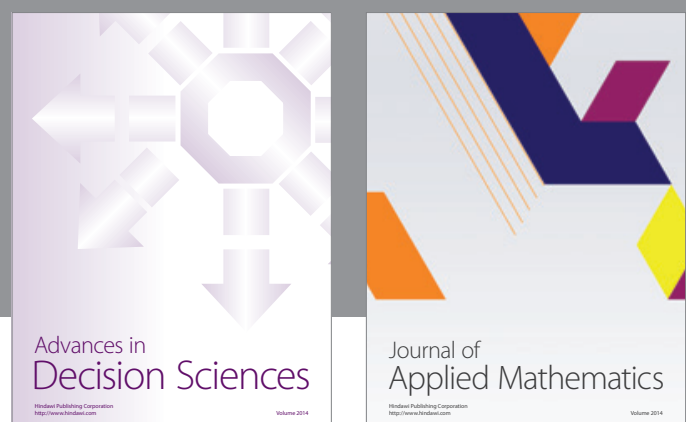

Journal of

Applied Mathematics
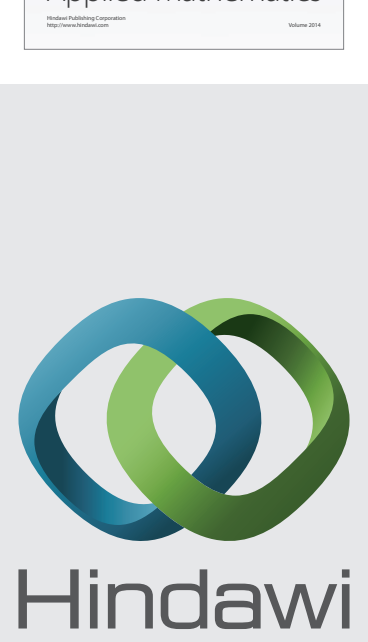

Submit your manuscripts at http://www.hindawi.com
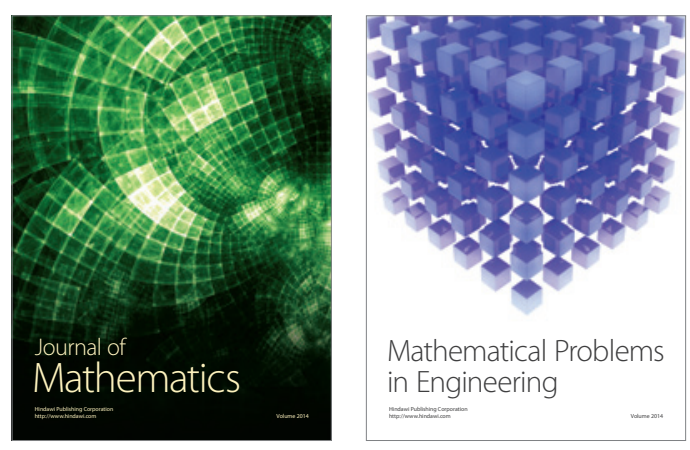

Mathematical Problems in Engineering
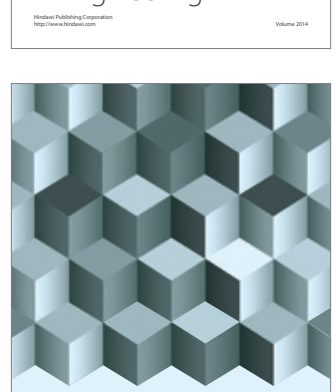

Journal of

Function Spaces
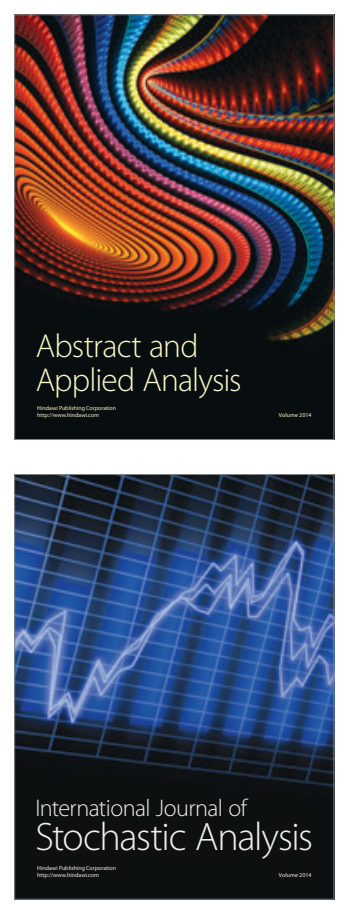

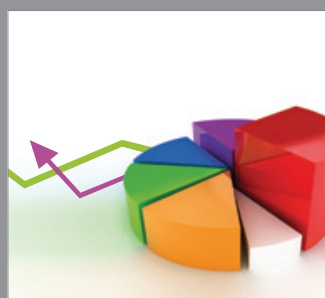

ournal of

Probability and Statistics

Promensencen
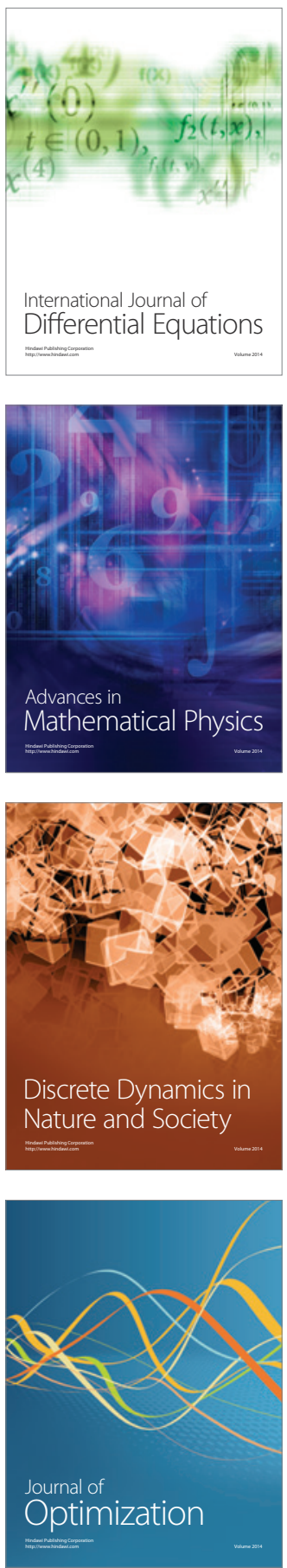